Revista de Comunicación y Salud, 2018, Vol. 8, no 1, pp. 45-59

Editado por Cátedra de Comunicación y Salud

ISSN: 2173-1675

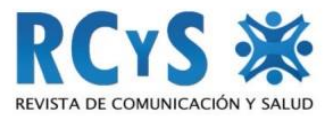

Enviado 17 de mayo de 2018

Aprobado 4 de julio de 2018

\title{
IMPACTO DEL SOPORTE ORGANIZACIONAL PERCIBIDO Y EL COMPROMISO ORGANIZACIONAL EN EL BALANCE VIDA-TRABAJO EN LA INDUSTRIA MANUFACTURERA DE EXPORTACIÓN GRANDE EN HERMOSILLO SONORA
}

\begin{abstract}
Impact of Perceived Organizational Support and Organizational Commitment in the Life-Work Balance in the Large Export Manufacturing Industry in Hermosillo Sonora
\end{abstract}

Ana Bolena Sotelo Medina

Universidad de Sonora - México

\section{Resumen}

El presente estudio tiene como objetivo determinar el impacto del soporte organizacional percibido a través de sus principales dimensiones que son el bienestar, satisfacción de necesidades y el reconocimiento. Tomando en cuenta que la organización considera una inversión que ayuda a mejorar las condiciones del trabajador a través de diferentes iniciativas para incrementar, mejorar y mantener en los trabajadores y al mismo tiempo su productividad. El bienestar, la satisfacción de necesidades y el reconocimiento también son medidos a partir de una metodología de corte cuantitativa, mediante la técnica de encuesta la cual mide el soporte organizacional percibido a través de las iniciativas que presenta cada empresa para lograr sus objetivos. Estas iniciativas se basan en los apoyos y esfuerzos extras que hacen las compañías y representan una importante inversión a nivel organización y posteriormente en la vida laboral. Es importante conocer qué tipo de apoyos que recibe el trabajador de su compañía y así valorar cuáles son aquellos con mayor impacto y efectividad. El compromiso organizacional, determinará el nivel de identificación que el empleado tiene hacia su organización desde sus componentes afectivos, de continuidad y normativos. En esta medida, se busca determinar el impacto en el Balance Vida-Trabajo a través del conflicto o el enriquecimiento que genera en los empleados operativos incluyendo hombres y mujeres que cuenten con una antigüedad mínima de 6 meses laborando en la Industria Manufacturera de Exportación catalogada como grande, son las empresas que tienen 251 empleados o más en la ciudad de Hermosillo, Sonora México.

Palabras clave: soporte organizacional percibido, balance vida-trabajo, compromiso organizacional, bienestar. 
Impacto del soporte organizacional percibido y el compromiso organizacional en el balance vida-trabajo en la industria manufacturera de exportación grande en Hermosillo, Sonora

\begin{abstract}
The objective of the study is to determine the impact of perceived organizational support through well-being, satisfaction of needs and recognition. The organization considers a investment that helps to improve the conditions of the worker through different initiatives to increase, improve and maintain the workers and at the same time their productivity. The well-being, satisfaction of needs and recognition are also measured from a quantitative methodology, using the survey technique which measures the perceived organizational support through the initiatives presented by each company to achieve its objectives. These initiatives are based on the extra support and efforts made by the companies and represent an important investment at the organization level and later in the working life. It is important to know if the workers receive support from the company and thus assess which are those with greater impact and effectiveness. The organizational commitment will determine the level of identification that the employee has towards their organization from its affective, continuity and normative components. In this measure, we seek to determine the impact on the Work-Life Balance through conflict or the enrichment that it generates in the operational employees, including man and woman with a minimum work experience in the company of six months of the Export Manufacturing Industry cataloged as large that have 251 employees or more in the city of Hermosillo, Sonora Mexico.
\end{abstract}

Keywords: perceived organizational support, work-life balance, organizational commitment, well-being.

\title{
Cómo citar el artículo
}

Sotelo Medina, A. B. (2018). Impacto del soporte organizacional percibido y el compromiso organizacional en el balance vida-trabajo en la industria manufacturera de exportación grande en Hermosillo, Sonora. Revista de Comunicación y Salud, 8(1), pp. 45-59. DOI: http://doi.org/10.35669/revistadecomunicacionysalud.2018.8(1).45-59

\section{INTRODUCCIÓN}

El trabajo es el medio por el cual satisfacemos nuestras necesidades básicas, todos tenemos una relación y contacto muy estrecho con todo lo relacionado al trabajo, el mundo laboral, sus efectos, sus beneficios, esfuerzos, bondades etc. Generalmente estamos enfocados en los aspectos negativos y nos gustaría cambiar, mejorar o empezar de nuevo con nuestra relación hacia el trabajo, de esta forma debemos atender la necesidad de cambiar nuestra visión o perspectiva y adoptar practicas más sanas, que proporcionen mayor satisfacción personal, mejores oportunidades de crecimiento, de balance vida-trabajo y bienestar laboral, físico, mental y al mismo tiempo estas prácticas beneficien de igual forma a la organización.

Para lograr medir e implementar de manera eficiente el soporte organizacional, compromiso organizacional y balance vida-trabajo, su impacto en el individuo en el aspecto físico-mental, social y económico es necesario entender sus inicios conceptuales a través de nuestra historia, usos y prácticas en el trabajo, sus 
Impacto del soporte organizacional percibido y el compromiso organizacional en el balance vida-trabajo en la industria manufacturera de exportación grande en Hermosillo, Sonora

consecuencias, cambios, avances, nuevas prácticas y modelos empleados en la actualidad, así como su justificación y efectividad en los diferentes aspectos en el individuo, en la sociedad y en el ámbito laboral.

Determinar la relación existente entre las prácticas de Balance Vida-Trabajo, en los trabajadores a través de las variables que influyen en la organización por medio de la implementación de prácticas administrativas, de comunicación efectiva, y a través de la disminución del conflicto y por consecuencia aumento en el enriquecimiento, demostrará empíricamente la influencia del soporte organizacional, su percepción y su trascendencia para el óptimo desarrollo operacional del lugar de trabajo y de los empleados, así como el compromiso organizacional que los trabajadores desarrollan hacia la organización y su impacto. La comunicación organizacional tiene un papel fundamental en estos procesos de comunicación interna desde los altos mandos, medios hasta llegar al empleado operativo y viceversa, considerando todos los factores que lleven a una mejora a nivel organización y en el trabajador siendo ambos de igual relevancia para la productividad y la satisfacción tanto organizacional como laboral y personal.

Hacia 1998 la Organización Mundial de la Salud define el concepto de salud como: estado de bienestar completo: físico, mental y social. También, define que el bienestar es un conjunto de constructos: competencia, autonomía, aspiración e integración funcional. Los trabajadores constituyen la mitad de la población del mundo y son los máximos contribuyentes al desarrollo económico y social. Su salud no está condicionada sólo por los peligros en el lugar de trabajo, sino también por factores sociales e individuales y por el acceso a los servicios de salud. Los riesgos para la salud en el lugar de trabajo, incluidos el calor, el ruido, el polvo, los productos químicos peligrosos, las máquinas inseguras y el estrés psicosocial provocan enfermedades ocupacionales y pueden agravar otros problemas de salud (OMS, 2017).

Las condiciones de empleo, la ocupación y la posición en la jerarquía del lugar de trabajo también afectan a la salud. Las personas que trabajan bajo presión o en condiciones de empleo precarias son propensas a fumar más, realizar menos actividad física y tener una dieta poco saludable. Además de la atención sanitaria general, todos los trabajadores y particularmente los de profesiones de alto riesgo, necesitan servicios de salud que evalúen y reduzcan la exposición a riesgos ocupacionales, así como servicios de vigilancia médica para la detección precoz de enfermedades y traumatismos ocupacionales y relacionados con el trabajo. Así, es importante destacar que la salud de los trabajadores es un requisito fundamental de la productividad y el desarrollo económico (OMS, 2017).

El plan de acción mundial de la OMS 2008-2017, está basado en algunos principios comunes. Todos los trabajadores deben poder disfrutar del más alto nivel posible de salud física y mental y de condiciones de trabajo favorables. El lugar de trabajo no debe perjudicar la salud ni el bienestar. La prevención primaria de los peligros para la salud de los trabajadores merece una atención prioritaria. Todos los componentes de los

Revista de Comunicación y Salud, 2018, Vol. 8, no 1, pp. 45-59 
Impacto del soporte organizacional percibido y el compromiso organizacional en el balance vida-trabajo en la industria manufacturera de exportación grande en Hermosillo, Sonora

sistemas de salud deben tenerse en cuenta a la hora de dar una respuesta integrada a las necesidades de salud concretas de la población activa.

El lugar de trabajo también puede servir de marco para llevar a cabo otras intervenciones esenciales de salud pública y promover la salud. Las actividades relacionadas con la salud de los trabajadores se deben planificar, poner en práctica y evaluar, con miras a reducir las desigualdades dentro de los países y entre ellos en lo que respecta a la salud de los trabajadores. Además, en esas actividades deben participar trabajadores y empleadores y sus representantes (OMS, 2007).

Un trabajo saludable es aquel en el que las presiones sobre los empleados son apropiadas en relación con sus capacidades y recursos, con la cantidad de control que tienen sobre su trabajo y con el apoyo que reciben de personas más cercanas. Dado que la salud no es simplemente la ausencia de enfermedad o dolencia, sino un estado positivo de completo bienestar físico, mental y social (OMS, 1986), un ambiente de trabajo sano es aquel en el que no sólo hay ausencia de condiciones perjudiciales, sino en el cual abunda la promoción de la salud. Estos pueden incluir la evaluación continua de los riesgos para la salud, la provisión de información y capacitación apropiada sobre temas de salud y la disponibilidad de prácticas y estructuras de apoyo organizacional que promuevan la salud. Un ambiente de trabajo saludable es aquel en el que el personal ha hecho de la salud y la promoción de la salud una prioridad y parte de su vida laboral.

En 1980 empezaron las investigaciones entre el lugar de trabajo y las características familiares que contribuyen al conflicto (Grenchas y Beutell, 1985). Para 1990, la salud de los trabajadores y las organizaciones eran tópico y hacían principal hincapié en el balance vida-trabajo y en el desarrollo de lugares de trabajo con prácticas sanas (Ilgen 1990 y Zedeck, 1992).

Estudios han demostrado, basados en décadas de investigación que el balance vidatrabajo es un componente crítico en los lugares de trabajo psicológicamente sanos (Hammer y Zimmerman, 2011). Los pasados 12 años, el término Balance Vida-Trabajo ha ido incrementando su frecuencia de uso (Kossek, Baltes y Matthews, 2011), es importante mencionar que la mayor parte de los trabajos empíricos han sido dirigidos hacia la Familia-Trabajo, con el fin de proveer el balance vida-trabajo a todos los empleados. El concepto de balance familia-trabajo es dominado por la palabra conflicto y a su vez con balance positivo familia-trabajo integrando conceptos como extensión, enriquecimiento y facilitación. El concepto de conflicto familia-trabajo se define como las demandas del trabajo y los roles familiares que son mutuamente incompatibles (Greenhaus y Beutell, 1985).

Greenhaus y Beutell (1985), proponen tres aspectos que conforman el conflicto familia-trabajo que son: tiempo, tensión y comportamientos. El conflicto del tiempo está en función de cuando un rol restringe la cantidad de tiempo que puede ser dedicado al otro rol, que derivan en el número de horas de trabajo a la semana, poca flexibilidad en el horario laboral y el número y edad de las personas menores dependientes en el

Revista de Comunicación y Salud, 2018, Vol. 8, no 1, pp. 45-59 
Impacto del soporte organizacional percibido y el compromiso organizacional en el balance vida-trabajo en la industria manufacturera de exportación grande en Hermosillo, Sonora

hogar. La tensión y el conflicto que genera está directamente relacionado con la afectación que genera en un desempeño satisfactorio de un rol sobre el otro, este se refleja en ambigüedad, supervisión y soporte pobre, desacuerdos familiares sobre el rol de géneros y la ausencia del soporte familiar. Por último, el conflicto en el comportamiento surge cuando las actitudes o comportamientos en un rol son incompatibles con las del otro rol. Según Greenhaus y Beutell, estas experiencias serán estresantes solo en el grado en el cual el individuo experiencia consecuencias negativas por no cumplir con las demandas de su rol.

El lado positivo antes mencionado dirige a la integración de lugares de trabajo con prácticas sanas e incluyen la extensión, enriquecimiento y facilitación. La extensión se define como la transferencia positiva y balance de afecto, habilidades, comportamientos y valores desde la fuente que origina hasta quien recibe, provocando en quien recibe efectos benéficos (Edwards y Rothbard, 2000; Hanson, Hammer y Colton, 2006). El enriquecimiento ocurre cuando las habilidades, el capital social (refiriéndose a quien se es y quien se quiere llegar a ser) y la flexibilidad o el efecto positivo es generado en uno de los roles, como la familia y contribuye a mejorar la calidad de vida en el otro rol, como el trabajo. El enriquecimiento familia-trabajo se definido como el grado en el que las experiencias de un rol mejoran o afectan positivamente la calidad de vida o el desempeño en el otro rol (Greenhaus y Powell, 2006). Por último, la facilitación se refiere a la participación de los individuos en uno aspecto en particular, como ejemplo la familia, provee mejoras, desarrollo, afecto, capital o eficiencia que contribuyen a mejorar el funcionamiento en el otro rol o aspecto de la vida como sería el trabajo (Wayne, Grzywacz, Carlson y Kacmar, 2007). Cada uno de estos constructos se identifica como bidireccional, en el cual el tiempo libre fuera del trabajo puede influenciar en el trabajo y de la misma forma el trabajo puede influenciar en el tiempo libre.

El 2011 Greenhaus y Allen introducen el concepto Balance Vida-Trabajo y lo definen como "un acercamiento general en el que la efectividad del individuo, la satisfacción laboral y las funciones o roles familiares son coherentes con sus valores de vida en un tiempo determinado" (p. 174). Es importante reconocer que el concepto balance puede malinterpretarse de acuerdo a la realidad de las familias trabajadoras quienes raramente logran un balance entre el trabajo y el tiempo libre fuera del trabajo. Esta definición surge de la perspectiva de un ambiente-persona-adecuado, en el cual los individuos evalúan su efectividad en los dos roles, familia y trabajo en cuanto a los estándares internos.

Lugares de trabajo con prácticas sanas son el resultado de la implementación de un grupo de políticas y prácticas a nivel organizacional diseñado para influenciar en el cambio de comportamiento a través de todos los niveles de la organización. Estas políticas y prácticas deben estar hechas a la medida, deben embonar en las necesidades particulares de la organización. Estas políticas y prácticas una vez que son implementadas de forma efectiva, a través de la comunicación sirven para dar forma a los comportamientos relacionados con la salud del empleado y fomentan un cambio de actitud (Hrebiniak, 2005; Lattin y Ramsey, 2009). De manera ideal estos

Revista de Comunicación y Salud, 2018, Vol. 8, oㅜ 1, pp. 45-59 
Impacto del soporte organizacional percibido y el compromiso organizacional en el balance vida-trabajo en la industria manufacturera de exportación grande en Hermosillo, Sonora

comportamientos sanos serán apoyados y reforzados por las características del ambiente, incluyendo el ambiente social de la organización, así como el ambiente físico. Con el paso del tiempo los empleados desarrollan y comparten interpretaciones comunes sobre un comportamiento sano y su importancia, lo que se espera y que este comportamiento es reconocido. La comunicación tiene un rol fundamental en participación integral, ya que alienta al cambio de comportamiento y ayuda a definir y establecer las percepciones deseadas.

La creación de políticas diseñadas para ayudar a los empleados a tener un balance entre su trabajo y su vida personal "Balance vida-trabajo" incluye iniciativas de horarios flexibles, teleconferencias, soporte con el cuidado de niños y adultos mayores, estas políticas son altamente valoradas por los empleados y se suma a los factores importantes del bienestar y de los ambientes laborales sanos (Perrewé, Treadway y Hall, 2003).

Kelloway, Hurrell y Day, (2008), argumentan que debemos enfocarnos en los esfuerzos positivos para incrementar el bienestar laboral, personal y administrativo. Literatura sobre los "Lugares Positivos de Trabajo" reconoce que la salud y los resultados positivos del empleado están influenciados no únicamente por una cultura laboral sana, y las iniciativas positivas que puedan imperar en el lugar de trabajo, sino por la congruencia en los valores individuales y organizacionales (Leiter, Frank y Matheson, 2009).

Según la Organización Mundial de la Salud en el 2015 y 2016, el estrés laboral provoca el 25 por ciento de los 75,000 infartos al año registrados en México; hasta ahora, el 75 por ciento de los trabajadores mexicanos padecen este mal, lo que coloca al país en primer nivel mundial en esta categoría (OMS, 2016). La Organización Internacional del Trabajo y la Organización Mundial de la Salud, indican que las causas del estrés laboral son: exceso o escasez de trabajo, horarios estrictos o inflexibles, inseguridad laboral, mala relaciones personales y falta de apoyo familiar (OIT, 2016; OMS, 2016).

El estrés laboral impacta en la economía pues datos de la OIT muestran que esta situación representa pérdidas de entre 0.5 y 3.5 por ciento del PIB de los países. En México el estrés produce mermas entre 5,000 y 40,000 millones de dólares al año. Los trastornos psicológicos derivados de las condiciones laborales que vive el trabajador repercuten de forma directa en el cuerpo, provocando enfermedades relacionadas con el estrés como: cardiopatía, trastornos digestivos, aumento en la tensión arterial, dolor de cabeza y trastornos músculo-esqueléticos. Además, que el estrés laboral trae como consecuencia aumento en la angustia o irritabilidad, dificultad en la toma de decisiones, cansancio, depresión, intranquilidad y falta de sueño (OIT, 2016).

Según la OCDE, México se sitúa por debajo del promedio en los temas de empleo y remuneración, estado de la salud, calidad medioambiental, vivienda, ingresos y patrimonio, sentido de comunidad, balance vida-trabajo, seguridad personal, satisfacción, y educación y competencias (OCDE, 2017).

Revista de Comunicación y Salud, 2018, Vol. 8, no 1, pp. 45-59 
Impacto del soporte organizacional percibido y el compromiso organizacional en el balance vida-trabajo en la industria manufacturera de exportación grande en Hermosillo, Sonora

En términos de empleo, cerca del $60 \%$ de las personas de entre 15 y 64 años de edad en México tienen un empleo remunerado, cifra menor que el promedio de la OCDE de $66 \%$. Cerca del $78 \%$ de los hombres tienen un empleo remunerado, en comparación con el $44 \%$ de las mujeres. En México el $28 \%$ de los empleados tienen un horario de trabajo muy largo, una de las cifras más altas de la OCDE, donde el promedio es de 13\%; y entre ellos, el $35 \%$ de los hombres trabajan muchas horas en comparación con el 17\% de las mujeres (OCDE, 2017).

Por otra parte, los factores que son detonantes del estrés son: injusticia laboral, incivilidad, conflictos vida-trabajo, mal liderazgo, poca flexibilidad, malas relaciones entre colegas, poco estimulo entre otros ya mencionados. Investigaciones indican que la implementación y las prácticas positivas contrarrestan los resultados negativos de los factores anteriormente mencionados, los cuales implican un cambio en las formas, cultura, participación y prácticas desde la alta dirección hasta los empleados. Algunas de las prácticas positivas son: Balance vida-trabajo, crecimiento y desarrollo personal, salud y seguridad, reconocimiento, sentido de pertenencia, satisfacción laboral, compromiso, salud física y mental, ambiente laboral, espacios físicos entre otros.

El Soporte Organizacional Percibido (POS) de Eisenberger, Huntington, Hutchinson y Sowa (1986), se mide la percepción de los empleados sobre como la organización valora su contribución y se preocupa por su bienestar. El soporte organizativo percibido surge con la observación de gerentes y su preocupación por el compromiso de sus empleados con la organización, los empleados se enfocan en el compromiso de la organización sostiene para que los empleados puedan satisfacer las necesidades de aprobación, estima y afiliación. POS incrementa la obligación de los empleados para ayudar a la organización a alcanzar sus objetivos, su compromiso afectivo con la organización y su expectativa de que el desempeño será recompensado.

Eisenberger y sus colegas (1986) desarrollaron el concepto de Soporte Organizacional Percibido (POS) para explicar el desarrollo del compromiso del empleado con una organización, propusieron que "los empleados desarrollen creencias globales sobre el grado en que la organización valora sus contribuciones y se preocupa por su bienestar" (1986 p. 501), y se refieren a esas creencias globales como el apoyo organizacional percibido.

En un marco de intercambio social, Eisenherger y sus colegas sostuvieron que tales creencias subyacen en las inferencias de los empleados sobre el compromiso de sus organizaciones con ellos (POS), lo que a su vez contribuye al compromiso de los empleados con sus organizaciones. Altos niveles de POS crean sentimientos de obligación, por lo que los empleados no sólo sienten que deberían comprometerse con sus empleadores, sino que también sienten la obligación de devolver el compromiso de los empleadores mediante la participación en comportamientos que refuerzan las metas de la organización. Es decir, los empleados buscan un equilibrio en sus relaciones de intercambio con las organizaciones por tener actitudes y comportamientos compatibles con el grado de compromiso del empleador con ellos como individuos. 
Impacto del soporte organizacional percibido y el compromiso organizacional en el balance vida-trabajo en la industria manufacturera de exportación grande en Hermosillo, Sonora

La investigación ha demostrado que el POS está positivamente relacionado con la conciencia en el desempeño de las responsabilidades laborales y el compromiso e innovación (Eisenberger, Fasolo y Davis-LaMastro, 1990). La percepción de ser valorados y cuidados por una organización también mejora la confianza de los empleados de que la organización cumplirá con sus obligaciones de cambio, de reconocimiento y de recompensar las actitudes y el comportamiento adecuado. Las recompensas pueden ser informales como un elogio o una tutoría, o formales como promociones y aumentos salariales.

La naturaleza del Compromiso Organizacional, analizado desde una perspectiva actitudinal y de comportamiento, la entendemos como: a) Compromiso actitudinal está enfocado en el proceso por el cual las personas piensan en su relación con la organización, el pensamiento en el cual los valores y metas del individuo son congruentes con los de la organización; b) Compromiso conductual por su parte, está relacionado al proceso de cada individuo de quedar inmerso dentro de la organización y la forma como llevan esta situación (Mowday et al.; 1982 p. 26).

La conceptualización del compromiso refleja tres principales componentes: a) el apego afectivo hacia la organización; b) los costos percibidos por dejar la organización y c) la obligación de continuar con la organización. Mowday et al. 1979, p. 226 describen el apego afectivo a la organización como "La fortaleza relativa de identificación que tiene cada individuo con la participación en una organización en particular". En referencia con los costos percibidos por dejar la organización, el individuo toma conciencia de la importancia que representa su pensión, jubilación, prestaciones entre otros aspectos que lo llevan a considerar su permanencia o continuidad en la organización. Kanter (1968) define este aspecto como "La ganancia asociada de continuar participando y el costo asociado por retirarse" (p. 504). Finalmente la obligación la describen Marsh y Mannari (1977) "Consideran moralmente correcto permanecer en la compañía independientemente del estatus o satisfacción que la empresa haya brindado a lo largo de tiempo" (p. 59).

Los conceptos relacionados anteriormente al compromiso organizacional, serán ahora referidos como: a) Affectivo; b) Continuidad y c) Normativo. El compromiso afectivo brevemente citado anteriormente hace referencia al apego emocional que el empleado tiene con la compañía, su identificación hacia ella y su nivel de integración con la organización, este tipo de empleados que tienen un fuerte apego efectivo hacia la organización, continúan trabajando porque quieren hacerlo, a diferencia de los empleados que tienen un compromiso de continuidad esto va en relación con la conciencia del costo asociado que representa el dejar la organización y continúan por necesidad dentro de su trabajo. Por último el compromiso normativo es el reflejo de un sentimiento de obligación de permanecer como empleado, este tipo de empleados sienten que deben permanecer con la organización (Meyer y Allen, 1991).

El compromiso afectivo está compuesto por cuatro diferentes categorías: características personales y estructurales, características relacionadas con el trabajo y la experiencia laboral (Mowday et al. 1982). El compromiso de continuidad pueden o no

Revista de Comunicación y Salud, 2018, Vol. 8, oㅜ 1, pp. 45-59 
Impacto del soporte organizacional percibido y el compromiso organizacional en el balance vida-trabajo en la industria manufacturera de exportación grande en Hermosillo, Sonora

estar relacionadas con el trabajo como la perder tiempo y esfuerzo adquiriendo habilidades no trasferibles, pérdida de beneficios atractivos, renunciar a su jubilación y/o pensión y los privilegios que representan o tener que interrumpir lazos familiares 0 relaciones personales se perciben como los costos potenciales por dejar de trabajar en alguna compañía en particular (Becker, 1960 citado por Meyer y Allen, 1991). El compromiso normativo sugiere que el sentimiento de obligación de permanecer en la compañía puede ser resultado de algún tipo de presión familiar, cultural, social antes de entrar a la organización. El compromiso normativo también se desarrolla cuando la compañía provee al empleado con "recompensas por adelantado" como el pago adelantado de algunos beneficios, becas, entrenamiento profesional etc. En ocasiones este tipo de inversiones que hace la compañía puede generar un desequilibrio entre la relación del empleado y la organización generando así el sentimiento de obligación, sentido de reciprocidad al comprometerse con la organización hasta el momento de saldar la deuda (Scholl, 1981).

Las consecuencias del compromiso como argumentan Katz (1964) y Organ (1987), que la efectividad organizacional depende no solo de mantener la fuerza laboral estable, los empleados deber realizar tareas confiadamente y estar dispuestos a comprometerse en actividades que van más allá de los requerimientos de sus labores, los empleados que quieren permanecer en la organización por un compromiso afectivo serán más aptos para realizar estas tareas o actividades que requieren un esfuerzo extra hacia la organización, que aquellos que necesitan permanecer o los que se sienten obligados a continuar en la organización.

El compromiso organizacional y su proceso referente al compromiso afectivo señala que los empleados que quieren permanecer en la organización que los provee con experiencias positivas y buscan su continuidad, son los más propensos a dar un extra siempre que contribuya a la efectividad de la organización, con el fin de mantener la equidad de acuerdo a la relación que tienen con la organización (Mowday et al. 1982).

Becker (1960) argumenta que los empleados cuyas habilidades se convierten menos competitivas pueden no experimentar el compromiso de continuidad hasta que o a menos que el mercado los obliga y se reconocen los costos de no hacer lo que se necesita para continuar en la compañía, es así como el compromiso de continuidad se desarrolla en algunos empleados.

El sentimiento de obligación de acuerdo a Weiner (1982) el sentimiento de obligación de permanencia en la organización se da como resultado de presiones normativas, las experiencias de socialización donde los valores, normas y creencias son la causa del sentimiento de obligación ya sea por ser un modelo a seguir dentro de la compañía, el uso de recompensas o restricciones. Generalmente los empleados que presentan un alto grado de lealtad dentro de la organización transmiten su lealtad por generaciones.

Es importante señalar que el concepto de reciprocidad para el compromiso normativo y de continuidad se identifican dentro del compromiso conductual, la diferencia reside en el motivo de reciprocidad, si este procede del compromiso afectivo entendemos que

Revista de Comunicación y Salud, 2018, Vol. 8, oㅜ 1, pp. 45-59 
Impacto del soporte organizacional percibido y el compromiso organizacional en el balance vida-trabajo en la industria manufacturera de exportación grande en Hermosillo, Sonora

se trata del deseo del empleado de contribuir al bienestar de la organización para mantener un nivel equitativo de beneficios mutuos, caso contrario si surge del compromiso normativo es derivado de la obligación de hacer lo que es correcto.

El compromiso organizacional está identificado como un factor importante en el entendimiento del comportamiento laboral de los empleados dentro de una organización. Para el desarrollo del instrumento de medición del compromiso organizacional se define compromiso organizacional como la fuerte identificación que un individuo tiene con una organización en particular y lo relacionan con tres factores: 1) una fuerte creencia y aceptación de los valores y metas que tiene la organización; 2) la disposición de hacer un esfuerzo personal extra a favor del bienestar de la organización y 3) un fuerte deseo de mantenerse como miembro de la organización (Porter y Smith, 1970). De esta definición se desprende que es más que lealtad a la organización, describe una activa relación con la organización, su deseo y disponibilidad de hacer esfuerzos extras para el bienestar de la compañía. Compromiso hace énfasis en el apego hacia la organización, incluyendo sus valores y metas a diferencia de la satisfacción laboral que solo enfatiza en el ambiente laboral en el cual un empleado desempeña sus tareas. Así el compromiso organizacional muestra más estabilidad en el tiempo que la satisfacción laboral (Mowday, Steers, y Porter, 1979).

En resumen se describe que el compromiso organizacional es un estado psicológico caracterizado por tres componentes principales: El deseo de mantenerse como miembro de una organización como resultado de experiencias laborales que generan sentimientos de confort y competencias positivas se conoce como compromiso afectivo. El que se refleja por la necesidad de permanencia debido al acto de traer a conciencia los costos relacionados con dejar el trabajo y la falta de alternativas se conoce como compromiso de continuidad y por último el tipo de compromiso que refleja obligación por una permanencia y sentido de lealtad y reciprocidad de favores que integran un compromiso económico del empleado a la organización se identifica como compromiso normativo.

El termino Balance Vida-Trabajo ha incrementado su frecuencia de uso en los pasados 10 años (Kossek, Baltes, y Matthews, 2011), con la extensión desde trabajofamilia a vida-trabajo, siendo más inclusivo con la vida personal fuera del lugar de trabajo, es decir, la vida fuera del trabajo (Fisher, Bulger, y Smith, 2009). El término Balance trabajo-familia está determinado por su interrelación con el conflicto entre el trabajo y la familia y por el derrame positivo, el enriquecimiento y facilitación que pueda surgir entre ambos. El conflicto trabajo-familia es un problema interno en el que las demandas del trabajo y los roles familiares son mutuamente incompatibles (Greenhaus y Beutell, 1985).

Recientemente se introduce el concepto Balance Vida-Trabajo, Greenhaus y Allen (2011) lo definen como: "Una evaluación general de la medida en que la efectividad y la satisfacción de los individuos en el trabajo y las funciones familiares son consistentes con sus valores de vida en un momento dado" (p. 174). El uso del término balance es criticado como una mala interpretación de la realidad de las

Revista de Comunicación y Salud, 2018, Vol. 8, oㅜ 1, pp. 45-59 
Impacto del soporte organizacional percibido y el compromiso organizacional en el balance vida-trabajo en la industria manufacturera de exportación grande en Hermosillo, Sonora

familias inmersas en el mundo laboral, quienes raramente logran tener este balance entre el trabajo y su vida fuera del trabajo. Esta definición surge de una perspectiva adecuada persona-ambiente, en la cual los individuos logran su efectividad en roles familiares y laborales en contra de los estándares internos familiares y laborales. Para lograr una mejor comprensión, se analiza la literatura referente a trabajo vida-familia, el cual a su vez está dominado por el concepto conflicto trabajo-familia. Debemos analizar la evolución de trabajo-familia para lograr el entendimiento y desarrollo del concepto balance vida-trabajo.

\section{OBJETIVOS E HIPÓTESIS}

El objetivo de la investigación es determinar el impacto del soporte organizacional percibido y el compromiso organizacional en el Balance Vida-Trabajo de los empleados operativos de la Industria Manufacturera de Exportación grande en Hermosillo, Sonora.

\section{Hipótesis:}

1. El Soporte Organizacional Percibido se relaciona directa y positivamente con el Balance Vida-Trabajo.

2. El Compromiso Organizacional se relaciona directa y positivamente con el Balance Vida-Trabajo.

3. El Compromiso Organizacional se relaciona directa y positivamente con el Soporte Organizacional Percibido.

\section{METODOLOGÍA}

Este estudio estará abordado desde una perspectiva interdisciplinar, donde la administración, la comunicación y la sociología serán las disciplinas que nos ayudan a comprender, describir, explicar y determinar las percepciones sobre el soporte organizacional percibido, el compromiso organizacional y el balance vida trabajo, desde el impacto económico, gerencial y sus resultados en individuo en productividad por citar un ejemplo. El papel fundamental de la comunicación en el ámbito laboral y personal, los conflictos y enriquecimiento que se presentan en todo proceso de integración administrativa, económica, política, social, económica, cultural y laboral.

Esta investigación es bajo el paradigma cuantitativo (Sampieri, 2007), se utilizará la recolección de datos fundamentada en la medición, posteriormente se llevará a cabo el análisis de los datos para dar respuesta a las preguntas de investigación, de esta manera se probarán las hipótesis establecidas previamente, confiando en la medición numérica, el conteo y en el uso de la estadística para intentar establecer con exactitud patrones en una población. Este enfoque funciona con reglas estrictas de lógica, verdad, leyes y predicciones.

La investigación propone una concepción global el trabajo, el soporte organizacional, el compromiso organizacional y el balance vida-trabajo, fundamentalmente orientado a

Revista de Comunicación y Salud, 2018, Vol. 8, no 1, pp. 45-59 
Impacto del soporte organizacional percibido y el compromiso organizacional en el balance vida-trabajo en la industria manufacturera de exportación grande en Hermosillo, Sonora

los resultados para explicar los fenómenos relacionados con el conflicto y el enriquecimiento, así como las diversas variables que componen el soporte organizacional. Se verificarán y comprobarán teorías por medio de estudios muéstrales representativos.

La investigación es de tipo correlacional, determinará si dos variables están correlacionadas o no y se realizará la propuesta de un modelo estructural de relación de las variables. Esto significa analizar si un aumento o disminución en una variable coincide con un aumento o disminución en la otra variable. Mediante la descripción de las características específicas y perfiles de los grupos que serán analizados, se pretende recoger y medir la información de manera conjunta. (Sampieri, 2007).

La investigación tiene un diseño no experimental de corte transversal para medir la prevalencia y efecto de la muestra poblacional en un solo momento temporal, permitiendo el estudio de diferentes variables, control en la selección de sujetos de estudio y proporcionando estimadores de prevalencia (Sampieri, 2007).

El Universo lo conforman las Industrias Manufactureras en Sonora, los datos oficiales son 382 empresas en el estado. La Secretaria de Economía del Gobierno del Estado divide en 9 sectores estratégicos que son: Aeronáutico/Aeroespacial, Automotriz, Minero, Eléctrico/Electrónico, Tecnologías de la Información, Agroindustrial, Energías renovables, Turístico y Biotecnologías y equipo médico. Existen más sectores fuera de la categoría que representan un número importante de empresas con relevancia para esta investigación.

La Población se representa por la Industria Manufacturera en Hermosillo, donde la muestra representativa es de 85 empresas dentro del Programa IMMEX, de las cuales, serán las empresas que cuenten con un número de empleados mayor a 251 y que sean empresas específicamente de Exportación.

El muestreo será no probabilístico intencional ya que se seleccionarán deliberadamente empresas en las cuales se observan y llevan a la práctica diaria acciones, principios, premisas y prácticas relacionadas con las variables que componen el soporte organizacional y el balance vida-trabajo. Como menciona Jiménez (1983) en el artículo "Población y muestra", cuando se realizan investigaciones descriptivas, transversales y no experimentales se aconseja una muestra de censo cuyo tamaño sea de 500 elementos, muestras que son utilizadas en ciencias como la sociología y la antropología, situación que se cubre al aplicarse un total de 100 cuestionarios en la fase de pilotaje. Jiménez (1983) califica este tipo de muestra como justificada cuando se requiere estudiar elementos excepcionales de cierta población, ya que es una de las formas de asegurarse de que éstos se incluirán en el estudio y por lo tanto se deberán elegir intencionalmente (Sánchez, 2013).

Revista de Comunicación y Salud, 2018, Vol. 8, no 1, pp. 45-59 
Impacto del soporte organizacional percibido y el compromiso organizacional en el balance vida-trabajo en la industria manufacturera de exportación grande en Hermosillo, Sonora

Se emplearan los instrumentos de Soporte Organizacional Percibido desarrollado por Eisenberger et al. (1986), Conflicto vida-trabajo desarrollado por Carlson et al., (2000), Enriquecimiento vida-trabajo desarrollado por Carlson et al., (2006) y el cuestionario de Compromiso Organizacional desarrollado por Mowday, Steers, y Porter, (1979).

\section{RESULTADOS}

Puesto a que se trata de una investigación que está en proceso de construcción, aún no se cuenta con resultados concluyentes. No obstsante puede decirse que, considerando que para reducir los niveles de conflicto en nuestra vida personal y profesional es necesario contar con un balance que mantenga de manera positiva productiva y conforme al desarrollo en los diferentes roles que desempeñar un trabajador, es conveniente adoptar prácticas que proporcionen el apoyo necesario para lograr objetivos en el ámbito personal y laboral. Esto, a través de la implementación de prácticas de soporte que deriven en un impacto positivo en el balance de vida-trabajo de todo individuo.

Una sociedad sana es una sociedad productiva y sin conflictos, el interés de esta investigación es precisamente lograr disminuir el conflicto en la vida laboral y en la vida fuera del trabajo, así como aumentar el enriquecimiento a través de prácticas de soporte organizacional y de compromiso organizacional que faciliten obtener este balance vida-trabajo, focalizados en mejorar nuestras condiciones de vida laborales y personales.

Lograr disminuir el ausentismo, la rotación, inseguridad laboral entre otros aspectos relevantes que afectan la productividad de las empresas es de gran importancia para el sector productivo. Si se implementan las prácticas de soporte organizacional y compromiso organizacional, el impacto positivo debe reflejarse no solo en la productividad de la empresa, también en la vida personal del empleado y por consiguiente en la sociedad.

\section{BIBLIOGRAFÍA}

Becker H. S. (1960). Notes on the concept of commitment. American Journal of Sociology, 66, 32-40.

Carlson, D. \& Kacmar, K. (2000). Work-Family Conflict in the Organization: Do life role values make a difference? Journal of Management, 26(5), 1031-1054.

Carlson, D., Kacmar, K., Holliday, J., \& Grzywacz, J. (2006). Measuring the positive side of the work-family interface: Development and validation of a work family enrichment scale. Journal of Vocational Behavior, 68, 131-164.

Edwards, J. R., \& Rothbard, N. P. (2000). Mechanisms linking work and family: Clarifying the relationship between work and family constructs. The Academy of Management Review, 25, 178-199.

Eisenherger. R., Huntington. R., Hutchison. S., \& Sowa, D. (1986). Perceived organizational support. Journal of Applied Psychology, 71, 500-507. 
Impacto del soporte organizacional percibido y el compromiso organizacional en el balance vida-trabajo en la industria manufacturera de exportación grande en Hermosillo, Sonora

Eisenberger, R., Fasolo, P., \& Davis-LaMastro, V. (1990). Perceived organizational support and employee diligence, commitment, and innovation. Journal of applied psychology, 75(1), 51.

Fisher, G. G., Bulger, C. A., \& Smith, C. S. (2009). Beyond work and family: A measure of work/ nonwork interference and enhancement. Journal of Occupational Health Psychology, 14, 441-456.

Greenhaus J. H., \& Allen T. (2011). Work family balance: A review and extension of the literature. In Tetrick, L. \& Quick, JC (Eds.), Handbook of occupational health psychology (2nd Ed.). (pp. 165-183). Washington, DC: American Psychological Association.

Greenhaus J. H., \& Beutell N. J. (1985). Sources and conflict between work and family roles. Academy of Management Review, 10, 76-88.

Greenhaus J. H., \& Powell G. N. (2006). When work and family are allies: A theory of work-family enrichment. Academy of Management Review, 31, 72-92.

Hammer, L. B., \& Zimmerman, K. L. (2011). Quality of work life. In S. Zedeck (Ed.), APA handbook of industrial and organizational psychology, Vol. 3, pp. 399-431. Washington, DC: American Psychological Association.

Hanson, G. C., Hammer, L. B., \& Colton, C. L. (2006). Development and validation of a multidimensional scale of perceived work-family positive spillover. Journal of Occupational Health Psychology, 11, 249-265.

Hrebiniak, L. G. (2005). Making Strategy Work: Leading Effective Execution and Change.

Ilgen, D. R. (1990). Health issues at work: Industrial and organizational psychology opportunities. American Psychologist, 45, 273-283.

Jimenéz Fernández, C. (1983). Población y muestra, El muestreo. En C. Jiménez Fernández, E. López Barajas, \& R. Pérez Juste, Pedagogía Experimental II. (Tomo I, págs. 229-258). Madrid: UNED.

Katz, D. (1964). The motivational basis of organizational behavior. Syst. Res., 9, 131146.

Kanter, R. (1968). Commitment and Social Organization: A Study of Commitment Mechanisms in Utopian Communities. American Sociological Review, 33(4), 499-517.

Kelloway, E. K., Hurrell Jr, J. J., \& Day, A. (2008). 20 Workplace interventions for occupational stress. The individual in the changing working life, 419.

Kossek, E. E., Baltes, B. B., \& Matthews, R. A. (2011). How work-family research can finally have an impact in organizations. Industrial and Organizational Psychology, 4, 352-369.

Leiter, M. P., Frank, E., \& Matheson, T. J. (2009). Demands, values, and burnout: Relevance for physicians. Canadian Family Physician, 55(12), 1224-1225.

Marsh, R., \& Mannari, H. (1977). Organizational Commitment and Turnover: A Prediction Study. Administrative Science Quarterly, 22(1), 57-75.

Meyer, J. P., \& Allen, N. J. (1991). A three-component conceptualization of organizational commitment. Human resource management review, 1(1), 61-89.

Mowday, R., Porter, L. and Steers, R. (1982). Employee-Organization Linkages: The Psychology of Commitment, Absenteeism, and Turnover. New York: Academic Press.

Mowday, R. T., Steers, M., \& Porter, L. W. (1979). The Measurement of Organizational Commitment. Journal of Vocational Behavior, 14, 224-247. 
Impacto del soporte organizacional percibido y el compromiso organizacional en el balance vida-trabajo en la industria manufacturera de exportación grande en Hermosillo, Sonora

OECD (2016), Panorama estadístico de la OCDE 2015-2016: Economía, medio ambiente y sociedad. México: Universidad Anáhuac.

OECD (2017). Balance vida-trabajo.Recuperado de http://www.oecdbetterlifeindex.org/countries/mexico/

OIT (2017). Estrés laboral en México. Recuperado de http://www.ilo.org

OMS (2016). Entornos laborales saludables. Recuperado de http://www.who.int/occupational health/evelyn hwp spanish.pdf

OMS (2017). Trabajo y salud en México. Recuperado de http://www.who.int/es/

OMS (2017). Plan de acción mundial. Recuperado de http://www.who.int/phe/publications/workers health global plan/es/

Organ, D. W., \& Konovsky, M. (1989). Cognitive versus affective determinants of organizational citizenship behavior. Journal of applied psychology, 74(1), 157.

Perrewé, P. L., Treadway, D.C., \& Hall, A. T. (2003). The work and family interface: Conflict, family-friendly policies, and employee well-being. In L. E. Tetrick \& D. A. Hoffman (Eds.), Health and safety in organizations: A multilevel perspective. New York: Jossey-Bass.

Porter, L. W., Crampon, W. J., \& Smith, F. J. (1972). Organizational commitment and managerial turnover: A longitudinal study (No. TR-13). California Univirvine graduate school of administration.

Sampieri, R. H., Collado, C. F., \& Lucio, P. B. (1996). Metodología de la investigación. Madrid: Edición McGraw-Hill.

Scholl, R.W. (1981) Differentiating Organizational Commitment from Expectancy as a Motivation Force. Academy of Management Review, 6, 589-599.

Wayne, J. H., Grzywacz, J. G., Carlson, D. S., \& Kacmar, K. M. (2007). Work-family facilitation: A theoretical explanation and model of primary antecedents and consequences. Human Resource Management Review, 17, 63-76.

Wiener, Y. (1982). Commitment in Organizations: A Normative View. The Academy of Management Review, 7(3), 418-428.

Zedeck, S. (1992). Introduction: Exploring the domain of work and family concerns. In S. Zedeck (Ed.), Frontiers of industrial and organizational psychology, Vol. 5; The Jossey-Bass management series and The Jossey-Bass social and behavioral science series. Work, families, and organizations (pp. 1-32). San Francisco, CA, US: JosseyBass. 